University of Nebraska - Lincoln

DigitalCommons@University of Nebraska - Lincoln

1989

\title{
PHYSICAL ASPECTS OF CHARGED PARTICLE TRACK STRUCTURE
}

\author{
R. H. Ritchie \\ Oak Ridge National Laboratory \\ R. N. Hamm \\ Oak Ridge National Laboratory \\ J. E. Turner \\ Oak Ridge National Laboratory \\ H. A. Wright \\ Oak Ridge National Laboratory \\ J. C. Ashley \\ Oak Ridge National Laboratory \\ See next page for additional authors
}

Follow this and additional works at: https://digitalcommons.unl.edu/usdoepub

Part of the Bioresource and Agricultural Engineering Commons

Ritchie, R. H.; Hamm, R. N.; Turner, J. E.; Wright, H. A.; Ashley, J. C.; and Banks, G. J., "PHYSICAL ASPECTS OF CHARGED PARTICLE TRACK STRUCTURE" (1989). US Department of Energy Publications. 91.

https://digitalcommons.unl.edu/usdoepub/91

This Article is brought to you for free and open access by the U.S. Department of Energy at DigitalCommons@University of Nebraska - Lincoln. It has been accepted for inclusion in US Department of Energy Publications by an authorized administrator of DigitalCommons@University of Nebraska - Lincoln. 


\section{Authors}

R. H. Ritchie, R. N. Hamm, J. E. Turner, H. A. Wright, J. C. Ashley, and G. J. Banks 


\title{
PHYSICAL ASPECTS OF CHARGED PARTICLE TRACK STRUCTURE*
}

\author{
R. H. Ritchie, $\uparrow$ R. N. Hamm, J. E. Turner, $\uparrow$ H. A. Wright, J. C. Ashley \\ Oak Ridge National Laboratory, Oak Ridge, Tennessee 37831-6123, U.S.A. \\ and \\ G. J. BASBAS \\ Physical Review Letters, Ridge, NY 11961, U.S.A.
}

(Received 21 February 1989)

\begin{abstract}
A plasmon generated by a swift charged particle constitutes a coherent excitation about the particle track. We discuss the physics of the plasmon in condensed matter and its effects on electron transport in liquid water. Criteria for the existence of the plasmon in a given substance, its characteristics when generated by a swift charged particle, its representation in impact parameter space, and its decay into localized excitations are described. We describe how plasmon excitation and decay are implemented in our Monte Carlo code, OREC, and how track structure calculations are affected by this mode.
\end{abstract}

\section{INTRODUCTION}

THE COMPLEXITY of phenomena associated with charged particle track formation in condensed media can hardly be overestimated. Even when restricting attention to purely physical events that occur in the early stages $\left(\sim 10^{-16}-10^{-13} \mathrm{~s}\right)$, one must still consider a wide range of possible mechanisms including e.g. (a) interactions involving collective, unlocalized excitations created in the medium, (b) geminate and volume recombination between electrons and ions, perhaps strongly affected by polarization of the medium, (c) Coulombic effects if the ionization density in the neighborhood of the track core is high, (d) migration of excitations in the medium, and (e) shakeoff, shakeup and Auger processes that may be affected strongly in the presence of condensed matter. These phenomena may occur in addition to the complicated interactions (such as energy degradation, electron multiplication, and recombination between electrons and ions liberated by the primary ionizing particle) that take place in dilute atomic or molecular assemblies.

In this paper, we review some of the work that has been done at Oak Ridge National Laboratory (ORNL) on "non-scaling" effects that are expected to operate in condensed matter. By this term we mean phenomena that cannot be adequately understood by accumulating information about radiation processes occurring in isolated molecules and then scaling these data according to density in order to predict what may happen in the condensed phase (Turner et al., 1983; Paretzke et al., 1986). Processes (a) to (e) mentioned above all fall into this category. Our approach has been to incorporate our best estimates of important non-scaling interactions into our Monte Carlo code "OREC", which simulates electron transport in liquid water, and to examine their manifestations through computer output in interesting cases.

One of the most striking differences between the dynamical response of dilute assemblies of molecules and the response of the same molecules in the condensed phase, lies in the possibility of coherent collective excitations of the latter. For example, swift charged particle energy losses in the range characteristic of valence electron excitations $(\sim 10 \mathrm{eV})$ may be initially unlocalized in regions $200 \AA$ in linear dimension relative to a nominal track center. Coherent excitations such as these may localize into small regions that are critical to the damage or inactivation of biologically interesting materials. In addition, the spatial and temporal scale of energy deposition in condensed media is such that one might expect interesting coherent interactions among the products of excitation and ionization. One of our motivations in looking at these possibilities stems from indications that bond breakages by ionizing radiations that are correlated over distances $\sim 10 \AA$ may be important in the killing or mutation of living cells.

\section{PLASMONS IN SOLIDS}

The plasmon is the quantum of electron density oscillation. Such oscillations have been studied in classical gas discharges since the early researches of Langmuir and coworkers (Tonks and Langmuir,

\footnotetext{
${ }^{*}$ Research sponsored by the Office of Health and Environmental Research, U.S. Department of Energy, under contract DE-AC05-840R21400, with Martin Marietta Energy Systems, Inc.

$\dagger$ Also Department of Physics, University of Tennessee, Knoxville, TN 37916, U.S.A.
} 
1929). Pines and Bohm (1952) realized that the plasmon must exist in condensed matter, and they developed a detailed quantal theory of its properties for the model system consisting of a collection of nearly free electrons moving in a uniform background of neutralizing positive charge (the electron gas). This model embodies some of the significant properties of the conduction electrons in metals. Their work, and that of succeeding researchers in this area, has been of great importance to the understanding of condensed matter.

\subsection{The electron gas model}

A simple classical derivation of the dynamics of plasma oscillations in the electron gas may be given. Consider a small displacement of the mobile electrons from their equilibrium positions at uniform density, $n_{o}$. Characterize this displacement by the vector function $\xi(\mathbf{r})$. Then the change in the density at $\mathbf{r}$ will be given by

$$
\delta n(\mathbf{r})=-n_{o} \nabla \cdot \xi
$$

Poisson's equation expresses the electric field that is created by this displacement as

$$
\nabla \cdot \mathbf{E}(\mathbf{r})=-4 \pi e \delta n(\mathbf{r})=4 \pi e n_{o} \nabla \cdot \boldsymbol{\xi} .
$$

Substituting from equation (1) into equation (2) and integrating, one finds that, to within an unimportant additive constant,

$$
\mathbf{E}(\mathbf{r})=4 \pi e n_{o} \xi .
$$

An electron, displaced by the amount $\boldsymbol{\xi}(\mathbf{r})$ from its equilibrium position, will experience a restoring force equal to $-e \mathbf{E}(\mathbf{r})$, and by Newton's second law its displacement will satisfy

$$
m \ddot{\xi}=-e \mathbf{E}(\mathbf{r})=-4 \pi n_{o} e^{2} \boldsymbol{\xi} .
$$

Taking the divergence of this equation and multiplying by $-n_{o}$, one finds that

$$
\delta \ddot{n}(\mathbf{r})+\omega_{p}^{2} \delta n(\mathbf{r})=0 .
$$

One sees that oscillations will occur at the plasma frequency

$$
\omega_{p}=\left(4 \pi n_{o} e^{2} / m\right)^{1 / 2},
$$

a well-known result. Note that these are fluctuations in the density that give rise to a restoring electric field directly opposing the displacement of the density. The electric field is thus said to be a longitudinal one, in contrast to the transverse character of the electric field of a light wave.

A somewhat more detailed, but still classical, model of the electron gas is furnished by the Bloch hydrodynamical approach (Bloch, 1933; Ritchie, 1957). Here one regards the assembly of electrons as a charged fluid that satisfies the equation of continuity, the Poisson equation and the Euler equation of fluid motion. Linearization of this set gives a true "wave equation" for the density fluctuation function, viz.,

$$
\left\{\partial^{2} / \partial t^{2}+\omega_{p}^{2}-\beta^{2} \nabla^{2}\right\} \delta n(\mathbf{r}, t)=0 .
$$

Now density fluctuations can propagate through the body of the electron gas with speed $\beta$, while oscilla. tions may occur at frequencies that are, in general, greater than $\omega_{p}$. One may see this by seeking a solution of equation (7) that describes a traveling wave of alternate compressions and rarefactions of density, with the form

$$
\delta n(\mathbf{r}, t)=\delta n_{o} \cos \left(\mathbf{k} \cdot \mathbf{r}-\omega_{k} t\right) .
$$

One finds, by substituting from equation (8) into equation (7), that $\omega_{k}=\left(\omega_{p}^{2}+\beta^{2} k^{2}\right)^{1 / 2}$ is the eigenfrequency of the wave with wavevector $\mathbf{k}$. It is longitudinal with an associated electric field that is parallel with $\mathbf{k}$.

In metallic systems, for which the description above is most appropriate, the plasmon energies are found to be $\sim 10 \mathrm{eV}$, from theory and experiment, while propagation speeds are $\sim 10^{8} \mathrm{~cm} / \mathrm{s}$.

\subsection{Plasmons in solids}

It is known that the plasmon is a well-defined quasi-particle in metals, semiconductors, insulators and liquids, but that because of damping processes it may correspond to quite broad resonance structures in the response functions of the respective media.

In order to illustrate some simple physical ideas associated with the concept of plasmons in a solid, consider the schematic diagram of Fig. 1. Assume that a wave of electron density fluctuations propagates through a system of atoms and that these fluctuations are made up of individual contributions due to displacements of the electron clouds about each atom. The wave is assumed to have a wavelength $\lambda$ much greater than the spacing between atoms. The displacement $\xi(x, t)$ of the electron clouds from their equilibrium positions is indicated schematically by the length of the small arrows drawn from the atom centers. The natural resonant frequency of each atom is taken to be $\omega_{o}$. The electric field amplitude $E$ associated with the wave is plotted vertically in the figure, though its direction is along the $x$-axis, i.e. it is longitudinal. If $n_{o}$ is the average electron density, the fluctuation in electron density $\delta n(x, t)$ is given by

$$
\delta n(x, t)=-n_{o} \partial \xi(x, t) / \partial x
$$

Assuming $\delta n(x, t)=\delta n_{k}(t) \exp (i k x)$, where the real part of all quantities is understood, the solution of Poisson's equation for the associated scalar electric potential $\phi(x, t)$

$$
\partial^{2} \phi / \partial^{2} x=4 \pi e \delta n(x, t)
$$

is $\phi=-4 \pi e \delta n_{k}(t) \exp (i k x) / k^{2}$, and

$$
E=-\partial \phi / \partial x=4 \pi i e \delta n_{k}(t) \exp (i k x) / k
$$

We evaluate the energy of the system by considering four separate contributions. The energy stored in the 


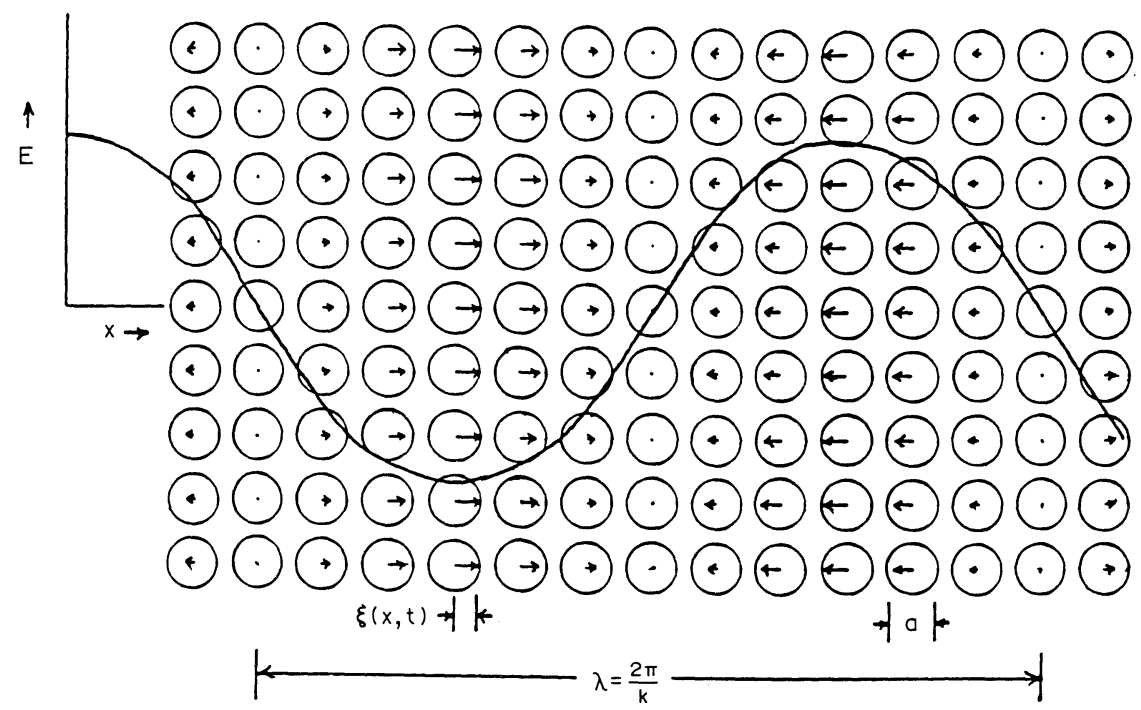

FIG. 1. A schematic representation of the polarization associated with a plasmon wave propagating through an assembly of atoms. The electric field amplitude $E$ is plotted vertically although its direction is in the direction of propagation. The small arrows indicate the displacement of the electron clouds of the atoms, which have resonant frequency $\omega_{o}$.

electric field per unit volume may be expressed as

$$
W_{\text {Coul }}=\left\langle|\operatorname{Re}(E)|^{2}\right\rangle / 8 \pi=(4 \pi e / k)^{2}\left|\delta n_{k}(t)\right|^{2} / 16 \pi,
$$

where an average over space is indicated by the angular brackets.

The kinetic energy per unit volume due to the displacement $\xi(x, t)$ is

$$
W_{\mathrm{KE}}=m n_{o}\left\langle|\operatorname{Re} \dot{\xi}|^{2}\right\rangle / 2=m\left|\delta \dot{n}_{k}\right|^{2} / 4 n_{o} k^{2}
$$

while the potential energy associated with the displacement of the electron clouds against their natural restoring forces, assumed to resonate at the natural frequency $\omega_{o}$, may be written

$$
W_{\text {res }}=m \omega_{o}^{2}\left\langle\left|\delta n_{k}(t)\right|^{2}\right\rangle / 4 n_{o} k^{2} .
$$

A phenomenological term describing an increase in velocity of the wave due to the compression of density may be taken proportional to $\delta v=v_{o} \delta n_{k}(t) / n_{o}$, where $v_{0}$ is a characteristic speed depending on the medium. This corresponds to the energy density

$$
W_{\text {vel }}=m v_{o}^{2}\left|\delta n_{k}\right|^{2} / 4 n_{o} .
$$

The total energy density is then

$$
W=m\left(\left|\delta \dot{n}_{k}\right|^{2}+\left(\omega_{o}^{2}+\omega_{p}^{2}+v_{o}^{2} k^{2}\right)\left|\delta n_{k}\right|^{2}\right) / 4 n_{o} k^{2} .
$$

This clearly shows that the wave propagates with eigenfrequency

$$
\omega_{p}=\left(\omega_{o}^{2}+\omega_{p}^{2}+v_{o}^{2} k^{2}\right)^{1 / 2} .
$$

The frequency of the wave is increased above the natural frequency of the constituent atoms due to Coulombic interactions among them. Of course, this simple model neglects chemical shifts of the atomic levels, and of the corresponding schematic atomic frequency $\omega_{0}$ used here, as the atoms are assembled to make up the solid. Nevertheless, upward shifts of plasmonic energies from the energies of the isolated constituent atoms are seen in experimental data on solids and liquids and may be attributed, at least in part, to collective effects. The $k$-proportional term in equation (17) represents only a qualitative trend, and must be established for each material.

\subsection{Dielectric theory}

The dielectric function codifies in an important way the dynamical properties of matter. The optical dielectric function has been determined for a large number of different materials over a fairly wide range of frequencies (Palik, 1985) and relates to the long wavelength behavior of these materials. This function, $\epsilon(\omega)=\epsilon_{1}(\omega)+i \epsilon_{2}(\omega)$, characterizes the response of a medium to very long-wavelength disturbances. Its frequency variation is determined by the spectrum and strength of electronic and molecular transitions.

Consider a classical point charge proceeding at constant velocity through a medium with response represented by the function $\epsilon_{k, \omega}$, where now the dielectric function depends on the wavenumber, $k$, as well as the frequency, $\omega$, of the disturbance. We denote $\epsilon_{o, \omega}$ by $\epsilon(\omega)$. To find the effect of this motion on the medium, it is useful again to solve the Poisson equation for $\phi(\mathbf{r}, t)$, the resulting scalar electric potential. This may be written

$$
\nabla^{2} \phi=-4 \pi Z e \delta^{3}(\mathbf{r}-\mathbf{v} t),
$$

where $Z e \delta^{3}(\mathbf{r}-\mathbf{v} t)$ is the charge density constituted by the moving charge. Expressing all functions as Fourier integrals of the form

$$
\begin{aligned}
f(\mathbf{r}, t)=(1 / 2 \pi)^{4} \int \mathrm{d}^{3} k \int \mathrm{d} \omega & \\
& \times \exp (i[\mathbf{k} \cdot \mathbf{r}-\omega t]) f_{\mathbf{k}, \omega},
\end{aligned}
$$


one finds, for the Fourier components of the electric potential, that

$$
\phi_{\mathbf{k}, \omega}=8 \pi^{2} Z e \delta(\omega-\mathbf{k} \cdot \mathbf{v}) /\left(k^{2} \epsilon_{k, \omega}\right) .
$$

If $\epsilon$ is a constant, $\epsilon_{o}$, one finds that

$$
\phi(\mathbf{r}, t)=Z e /|\mathbf{r}-\mathbf{v} t| \epsilon_{o},
$$

which, of course, is just the Coulomb potential of the charge screened by the constant dielectric response of the medium. This screening of the longitudinal electric field of a charge is a striking manifestation of the difference between the response of a medium to a photon and to a charge: the photon causes a transverse disturbance in the medium that does not accumulate charge. It is thus unscreened, but not unmodified, in its passage through the system. When the full variation of $\epsilon_{k, \omega}$ with $k$ and $\omega$ is included, charge screening is still present but may be strongly modified from that predicted by equation (20a).

To evaluate energy loss to the medium, one has only to evaluate the retarding force on the charge due to the electric field it induces; one finds that

$$
\begin{aligned}
-\mathrm{d} E / \mathrm{d} x=\left[\frac{Z e}{v}\right]^{2} & \frac{2}{\pi} \int_{0}^{\infty} \omega \mathrm{d} \omega \\
& \times \int_{\omega / v}^{\infty} \frac{\mathrm{d} k}{k} \operatorname{Im}\left(-1 / \epsilon_{k, \omega}\right) .
\end{aligned}
$$

This gives the energy loss per unit length of travel and, in principle, includes losses to all possible kinds of optically active excitations that the medium can support. The differential inverse mean free path for energy loss $\hbar \omega$ and momentum transfer $\hbar \mathbf{k}$ is found by dividing the integrand by $\hbar \omega$, viz.,

$$
\mathrm{d}^{2} \Lambda^{-1} / \mathrm{d} \omega \mathrm{d} k=\frac{2}{\pi \hbar}\left[\frac{Z e}{v}\right]^{2} \operatorname{Im}\left(-1 / \epsilon_{k, \omega}\right) / k .
$$

The quantity $\operatorname{Im}\left(-1 / \epsilon_{k, \omega}\right)$ is often referred to as the energy-loss function.

For comparison, note that the attenuation coefficient (the absorption probability per unit length) of a photon with energy $\hbar \omega$ traveling in this medium is given by

$$
\mu=\frac{2 \omega}{c} \operatorname{Im}\left\{[\epsilon(\omega)]^{1 / 2}\right\},
$$

showing no screening effect but exhibiting maxima in absorption at frequencies that correspond approximately to maxima in $\operatorname{Im}[\epsilon(\omega)]$.

\subsection{Plasmon resonances in the response functions of condensed matter}

The well-known Drude-Sellmeier formula (Rosenfeld, 1965) for the complex, long-wavelength dielectric constant of a medium may be written

$$
\epsilon(\omega)=1+\sum_{n} \frac{f_{n} \omega_{p}^{2}}{\omega_{n}^{2}-\omega^{2}-i \gamma_{n} \omega},
$$

neglecting local field effects for simplicity. Here $\omega_{p}$, the plasma frequency, is given by $\omega_{p}=\left(4 \pi n_{o} e^{2} / m\right)^{1 / 2}$, as above, except that $n_{o}$ is now the total density of electrons. Also $f_{n}$ is the oscillator strength of the $n$th transition with frequency $\omega_{n}, \gamma_{n}$ is an empirical damping rate, and the sum goes over all possible transitions.

Williams et al. (1975) have shown clearly how Coulomb interactions among electrons give rise to an upward shift in the resonant behavior of the energy. loss function $\operatorname{Im}[-1 / \epsilon(\omega)]$. They show that an approximate formula may be obtained from equation (24) for $\omega_{r n}$, the resonant frequency of the $n$th transition. It displays a collective shift in the resonant frequency of a molecule as it is assembled into the condensed state, and is given by

$$
\omega_{r n} \approx\left(f_{n} \omega_{p}^{2}+\omega_{n}^{2}\right)^{1 / 2},
$$

which agrees in form with equation (17) if $v_{o}$ is set equal to zero there. These authors also point out that $\operatorname{Im}[\epsilon(\omega)]$ shows resonances corresponding to singleparticle transitions in a medium, while resonances in $\operatorname{Im}[-1 / \epsilon(\omega)]$ may correspond to collective modifications of these transitions. They consider that the frequencies $\omega_{n}$ become so closely spaced that $f_{n} \rightarrow f\left(\omega_{n}\right)$ may be considered to be a continuous function of its argument, and they demonstrate that a condition for a nearly pure plasma resonance to occur is

$$
\omega_{p}^{2}\left|\frac{\mathrm{d} f}{\mathrm{~d}\left(\omega^{2}\right)}\right| \gg 1,
$$

which agrees with a criterion put forward by Fano $(1960 \mathrm{a}, \mathrm{b})$. However, they argue that this criterion is too stringent in that collective effects may be present in liquids and solids in various degrees even when it is not fully satisfied.

Ehrenreich and Philipp (1962) point out that it is not necessary that $\epsilon(\omega)=0$ for a resonance in $\operatorname{Im}[-1 / \epsilon(\omega)]$ to occur. They note that a maximum of $\operatorname{Im}(-1 / \epsilon)$ corresponds to a plasma resonance if $\epsilon_{1}$ and $\epsilon_{2}$, in the neighborhood of the peak, are (i) small compared with unity, (ii) approximately linearly varying with $\mathrm{d} \epsilon_{1} / \mathrm{d} \omega>0$ and $\mathrm{d} \epsilon_{2} / \mathrm{d} \omega<0$, and (iii) $\left(\omega / \epsilon_{1}\right) \mathrm{d} \epsilon_{1} / \mathrm{d} \omega$ and $\left(\omega / \epsilon_{2}\right) \mathrm{d} \epsilon_{2} / \mathrm{d} \omega \gg 1$.

Examples of plasmon resonances in several different media are given in Figs 2-8. Figure 2 shows one of the clearest examples of this phenomenon as it manifests itself for $\mathrm{Al}$ metal. Here the contribution from interband transitions at the resonant energy of $\sim 15 \mathrm{eV}$ in the energy-loss function is very small indeed, as seen in the smallness of $\epsilon_{2}(\omega)$ there. Figure 3 shows similar data for $\mathrm{Ag}$, where now a. sharp hybrid resonance appears at $\sim 3.5 \mathrm{eV}$. Here, all of the Ehrenreich-Philipp (E-P) criteria are satisfied, although an important role is played by the $d-s$ interband transition, strongly modified by the collective motion of the conduction electrons. In addition there is a broad resonance at $\sim 8 \mathrm{eV}$, which seems to arise mostly from collective interactions among the conduction electrons. Figure 4 displays 


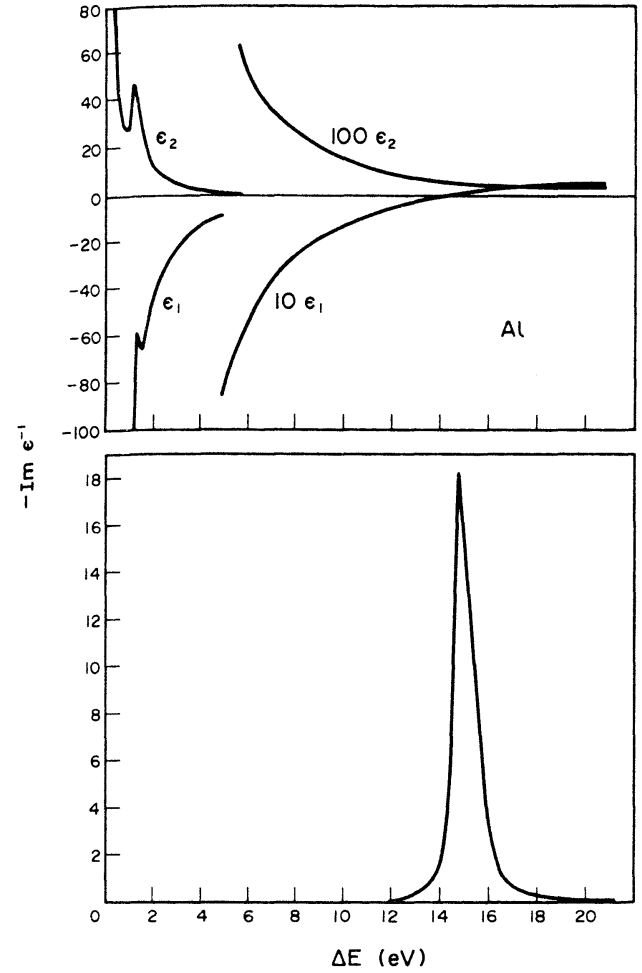

FIG. 2. The spectral dependence of the real and imaginary parts of the dielectric constant and the energy-loss function for Al metal calculated from optical data (from Raether, 1965).

the optical constants of the semiconductor silicon as they depend on frequency. Again the E-P conditions are well satisfied for the broad resonance at $\sim 17 \mathrm{eV}$, with essentially no single-particle component of the

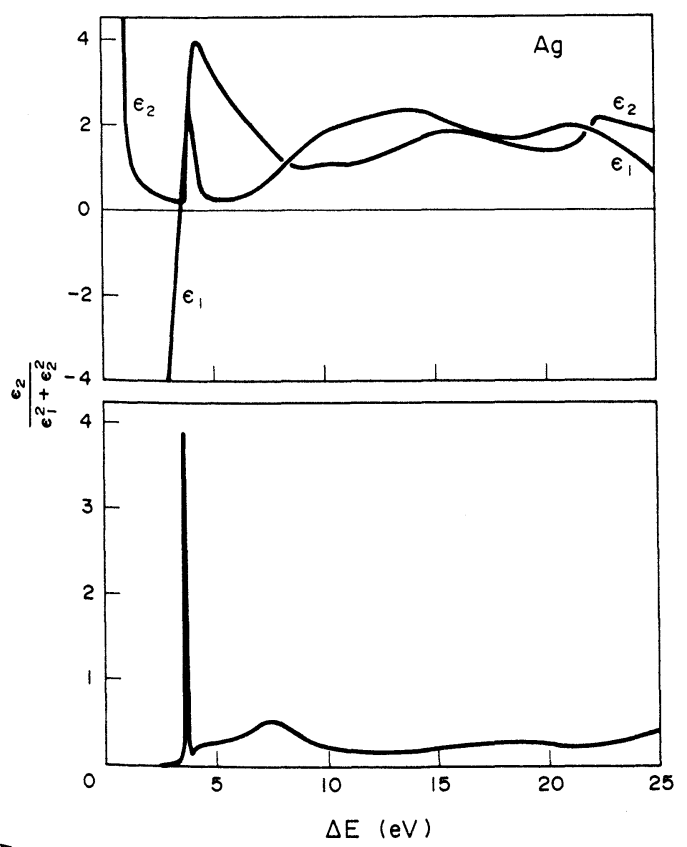

FIG. 3. The spectral dependence of the real and imaginary parts of the dielectric function of $\mathrm{Ag}$ metal and the energyloss function (from Raether, 1965).

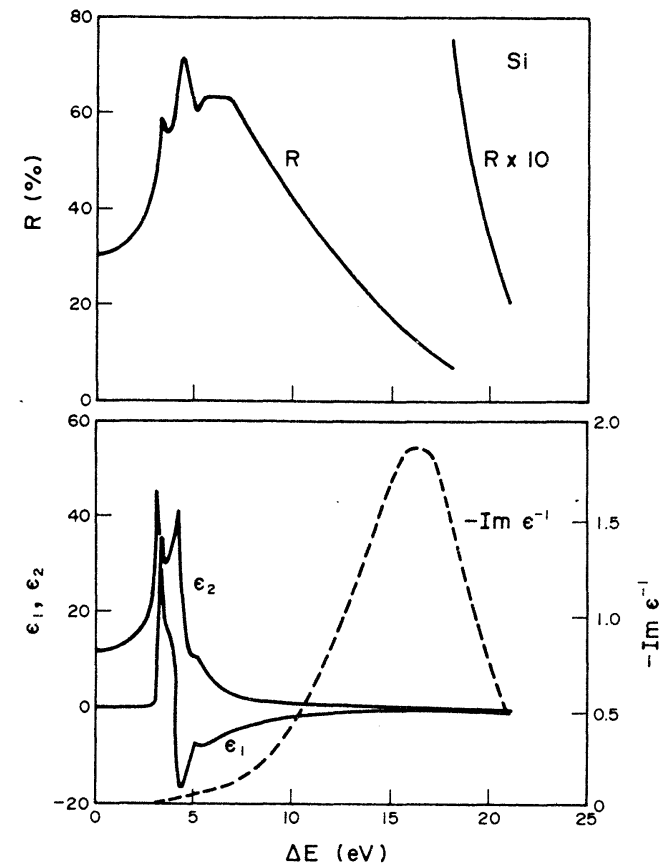

FIG. 4. The spectral dependence of the reflectance $R$, the real and the imaginary parts of the dielectric function and the energy-loss function for the semiconductor silicon (from Raether, 1965).

response in that collective state, as witnessed by the smallness of $\epsilon_{2}$ in that region.

In Fig. 5 the dielectric functions and the actual loss function determined from energy-loss measurements

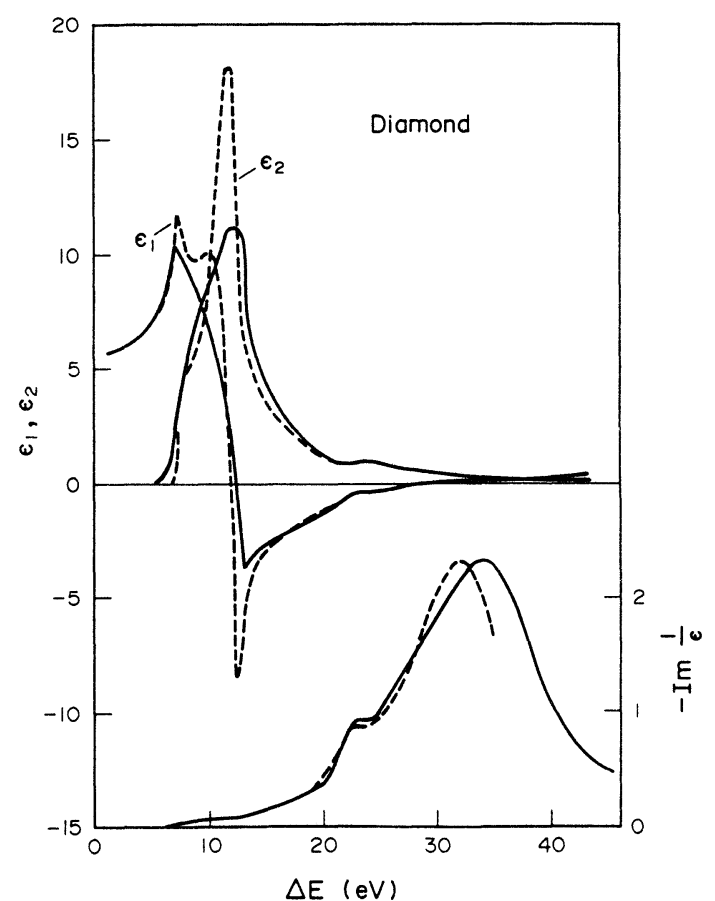

FIG. 5. The dielectric functions and the energy-loss function for diamond. The solid lines show data inferred from energy-loss measurements while the dotted lines are optical data (from Raether, 1965). 

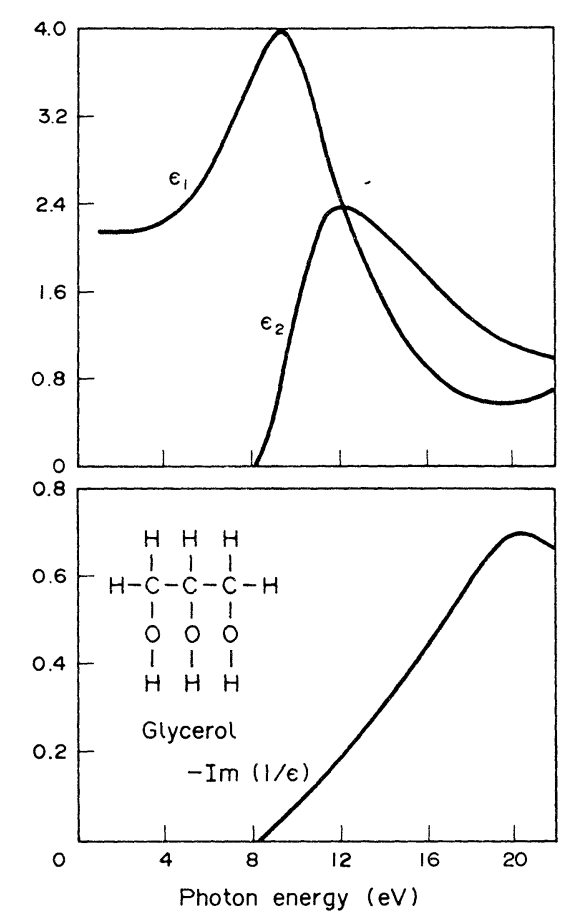

FIG. 6. Dielectric functions and energy-loss function for liquid glycerol (Williams et al., 1975).

of the hard insulator diamond are presented (Daniels et al., 1970). The evidence for a plasmonic resonance is very strong. The E-P criteria are well satisfied. It is worthwhile to note that there is a large energy gap in diamond and essentially no electrons in the conduction band, and little possibility that real interband transitions participate in this prominent resonance.

Williams et al. (1975) showed that plasmon-type resonances are exhibited in the optical response of several liquids that they had determined experimentally. Figure 6 shows the optical constants of glycerol as taken from their paper. One sees a broad maxi. mum in $\operatorname{Im}(-1 / \epsilon)$ that is shifted upward from the peak in $\epsilon_{2}(\omega)$, with the E-P criteria generally satisfied.

Figure 7 shows the optical constants of liquid wate as inferred from the data of Heller et al. (1974). Here the E-P criteria are all satisfied, except that the quantity defined in the last part of (iii) is comparable with unity. However, it is clear that a strong collective component is present in the response of this medium to charged particles. On this basis we have constructed the full response function of liquid water from the optical data using reasonable assumptions about the dispersion of $\operatorname{Im}(-1 / \epsilon)$ in $k-\omega$ space (Ritchie et al., 1978) and have employed algorithms described below to represent the decay of initially unlocalized coherent collective excitations created by swift electrons or ions.

\subsection{The dielectric function of the electron gas}

A convenient representation of the dielectric function of the electron gas has been given by Lindhard (1954) and modified by many workers. A much-used analytical modification that includes damping in a phenomenological way was proposed by Mermir (1970).

Figure 8(a) shows a plot of the energy-loss function of a damped electron gas as it depends on wavenumber $k$, and the energy $\hbar \omega$. The parameters assumed in computing these data were a damping rate of $3 \mathrm{eV}$ and a plasma energy of $15.4 \mathrm{eV}$. One sees a narrow peak that begins at the plasma energy fo: $k=0$ and increases in energy with increasing $k$. This corresponds to the relation $\omega_{k}=\left(\omega_{p}^{2}+\beta^{2} k^{2}\right)^{1 / 2}$ given above on the basis of the hydrodynamical model for small $k$ but deviates from this relation for larger $k$. The peak signals the possibility of plasmon gener-

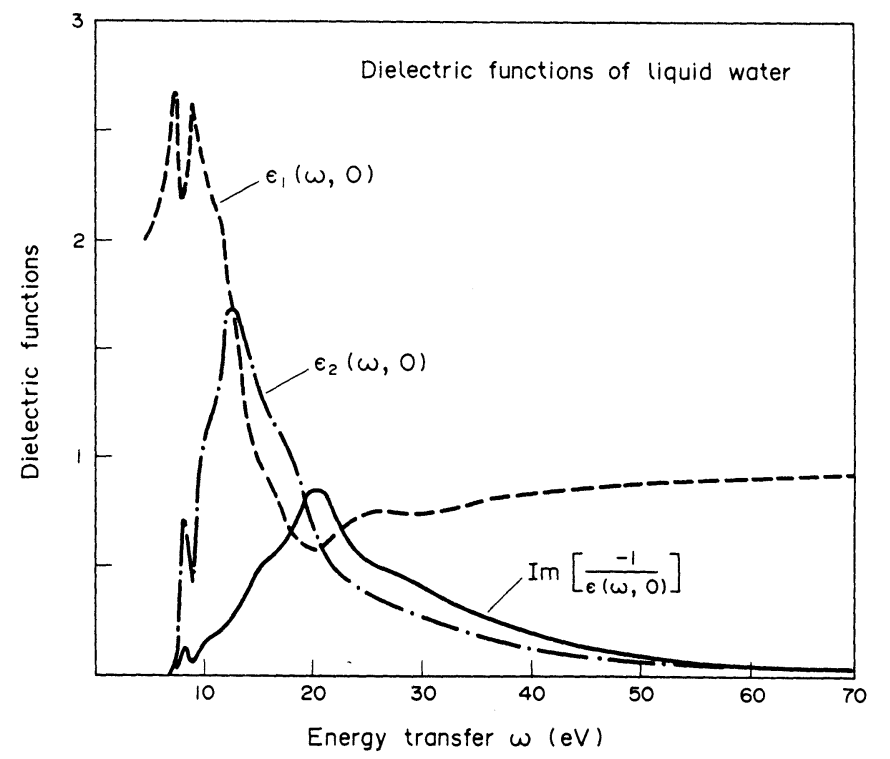

FIG. 7. Dielectric functions and energy-loss function for liquid water (Heller et al., 1974). 

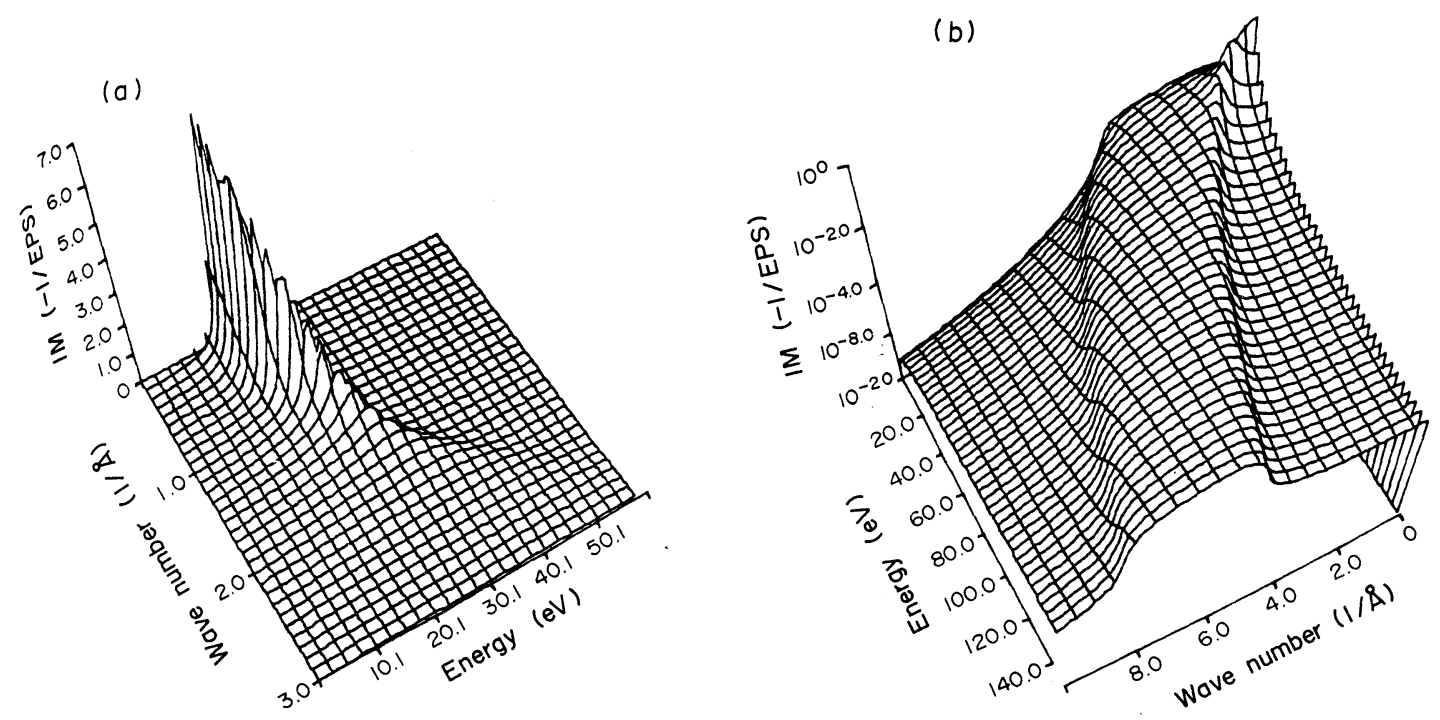

Fig. 8. (a) A contour plot of the energy-loss function of a damped electron gas as it depends on wavenumber $k$ in $\AA^{-1}$, and energy transfer $E$ in $\mathrm{eV}$. The damping constant was taken to be $3 \mathrm{eV}$ and the plasma energy $15.4 \mathrm{eV}$. (b) The same data but with $\operatorname{Im}\left(-1 / \epsilon_{k, \omega}\right)$ plotted on a logarithmic scale.

ation by an incident projectile. It merges into a wider region in the $k-\omega$ plane as $k$ increases and shows the effects of possible single-particle excitation from the occupied energy levels of the electron gas. Figure 8(a) is deceptive in that the plasma resonance appears to be overwhelmingly dominant compared with the single-particle effects; however, the latter are represented by a surface that extends over a wide range of the energy and wavenumber variables, while the plasma resonance effects are confined to a rather small region. This is illustrated by Fig. 8(b) which is the same quantity plotted on a logarithmic scale for $\operatorname{Im}(-1 / \epsilon)$ but viewed from another direction. Now one sees a region in the $k-\omega$ plane that moves upward in $\omega$ approximately as the square of $k$ and corresponds to the region in which electron-hole excitations dominate.

\subsection{The dielectric response function of a model solid}

Theoretical construction of the dielectric function of real crystalline solids requires considerable numerical work (Walter and Cohen, 1972; Sramek and Cohen, 1972) and in full representation, involves the fact that it is in reality a matrix, $\epsilon_{\mathbf{Q}, \mathbf{Q}^{\prime}}(k, \omega)$, where $\mathbf{Q}$ and $\mathbf{Q}^{\prime}$ are reciprocal lattice vectors in the crystal. Often one uses the approximation of Ehrenreich and Cohen (1959) in which the off-diagonal components of $\epsilon$ are neglected and the self-consistent field result

$$
\begin{aligned}
\epsilon_{\mathbf{k}, \omega}= & 1+\left(\frac{4 \pi \mathrm{e}^{2}}{\Omega k^{2}}\right) \sum_{\mathbf{q}} \sum_{l} \\
& \times \sum_{l} \frac{\left\langle\mathbf{q} l \mid \mathbf{q}+\mathbf{k}, l^{\prime}\right\rangle\left[f_{o}\left(E_{\mathbf{k}+\mathbf{q}, l^{\prime}}\right)-f_{o}\left(E_{\mathbf{q} /}\right)\right]}{E_{\mathbf{q} l}-E_{\mathbf{k}+\mathbf{q}, l^{\prime}}+\hbar \omega+i \eta}
\end{aligned}
$$

is used. Here $E_{\mathrm{q} l}$ is the one-electron energy of an electron in the $l$ th band and with wave vector $\mathbf{q}$, $\langle\mathbf{x} \mid \mathbf{q}, l\rangle=u_{\mathbf{q} l}(\mathbf{x}) \exp (\mathrm{iq} \cdot \mathbf{x}) / \Omega^{1 / 2}$ is the wave function in the reduced band scheme, $\Omega$ is a normalization volume, and $\eta$ is a positive infinitesimal.

Some time ago we (Ritchie et al., 1975; Tung et al., 1977) devised a simple analytical representation of the dielectric function of an insulator that displays band structure and predicts a plasmonic mode that is broadened due to single-particle effects. We assumed that the valence states of the model solid may be represented in the tight-binding approximation by simple localized orbitals. Conduction electrons are represented by plane waves orthogonalized to the valence states. After averaging over nuclear positions which, for simplicity, are assumed to be randomly distributed, one derives from the irreducible polarization propagator for the solid an analytical expression for the imaginary part of the dielectric function $\epsilon_{k, \omega}$. From the Kramers-Kronig relation one finds the complete response function, which we require to satisfy an important sum rule.

Figure 9 shows $\operatorname{Im}\left(-1 / \epsilon_{k, \omega}\right)$ for a tight-binding solid with a band gap of $9 \mathrm{eV}$, an atomic density of $4.05 \mathrm{~g} / \mathrm{cm}$ and an $s$-orbital radius of $0.78 \mathrm{a} . \mathrm{u}$. One sees clearly that a collective mode exists for small $k$ and that the damping of this mode becomes larger as $k$ increases. For comparison, the surface $\operatorname{Im}\left(\epsilon_{k, \omega}\right)$ shows monotonic decrease above the threshold as $\omega$ increases at constant $k$. The dispersion of the plasmon shown in Fig. 9 increases with increasing $k$, so that the response function surface finally goes into the "Bethe ridge", corresponding to asymptotic freeelectron-like behavior. This model has been used by our group to represent the response of several different condensed media to external probes (Ritchie et al., 1975; Ashley and Anderson, 1981; Ashley, 1988). 


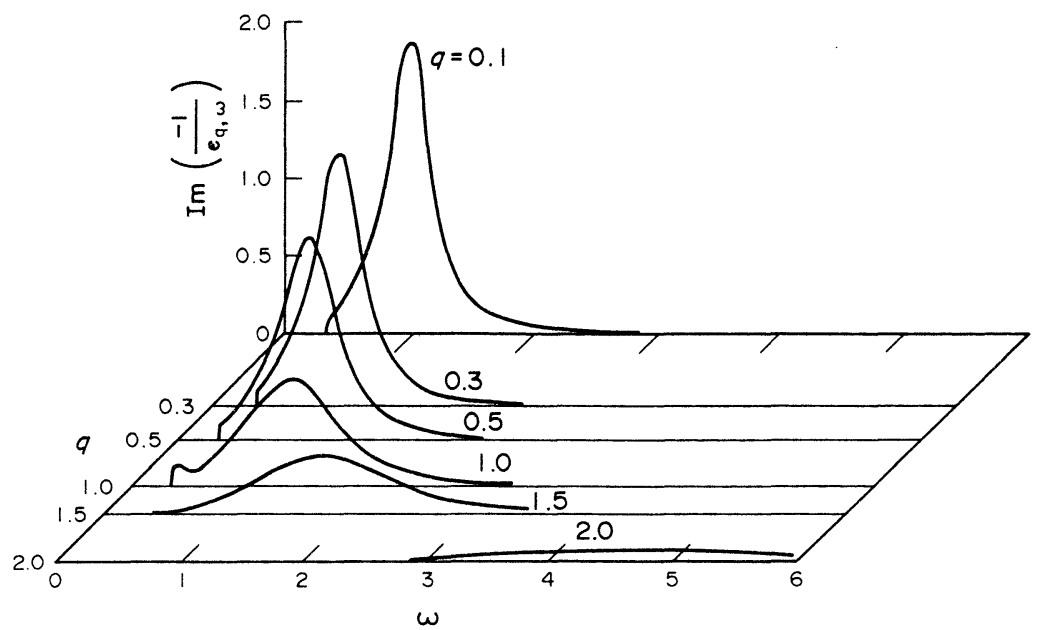

FIG. 9. The energy-loss function for a tight-binding solid with a band gap of $9 \mathrm{eV}$, an atomic density of $4.05 \mathrm{~g} / \mathrm{cm}$ and an orbital radius of $0.78 \mathrm{a} . \mathrm{u}$. (Ritchie et al., 1975).

\section{IMPACT PARAMETER REPRESENTATION}

\subsection{Introduction}

In quantal collision theories, momentum and energy are usually taken to be good quantal variables (Bethe, 1930). Classical collision theory, on the other hand, uses position and time to describe interactions between a probe and a target (Bohr, 1913, 1948). In modern physics, one may wish to express quantal theories in terms of space-like variables. For example, experiments are now common in which one measures, by means of a narrowly focused beam of swift electrons, the distribution in energy of losses experienced in a very small region of space. Also, in experiments with channeled ions, and in microdosimetry, one is interested in the spatial coherence of unlocalized excitations created by swift ions and electrons, and their ultimate localization through transfer of energy to, e.g. single-particle excitations.

Figure 10 is taken from Bohr (1948), illustrating schematically the passage of a charged particle with speed $v$ through an assembly of atoms each con- sidered to resonate at the frequency $\omega_{0}$. The arrows indicate displacements of the center of charge of each atom under the perturbing influence of the particle. A measure of the part of the medium most strongly influenced is indicated by the dashed line roughly defining a cone about the ion trajectory. An atom at impact parameter $b$ from the track experiences a time-varying force that, if screening by other atoms is neglected, has a distribution characterized by the fundamental frequency

$$
\omega_{\text {fund }}=v / b \text {. }
$$

Those atoms experiencing forces that vary slowly enough so that $\omega_{\text {fund }} \ll \omega_{o}$ will respond adiabatically to these forces and absorb essentially no energy from the particle. A measure of the region in impact parameter space inside which collisions deliver appreciable energy to the medium is then given by the Bohr cutoff value

$$
b_{c}=v / \omega_{o} .
$$

This quantity may be as large as several hundred angstroms for the most loosely bound atoms in some

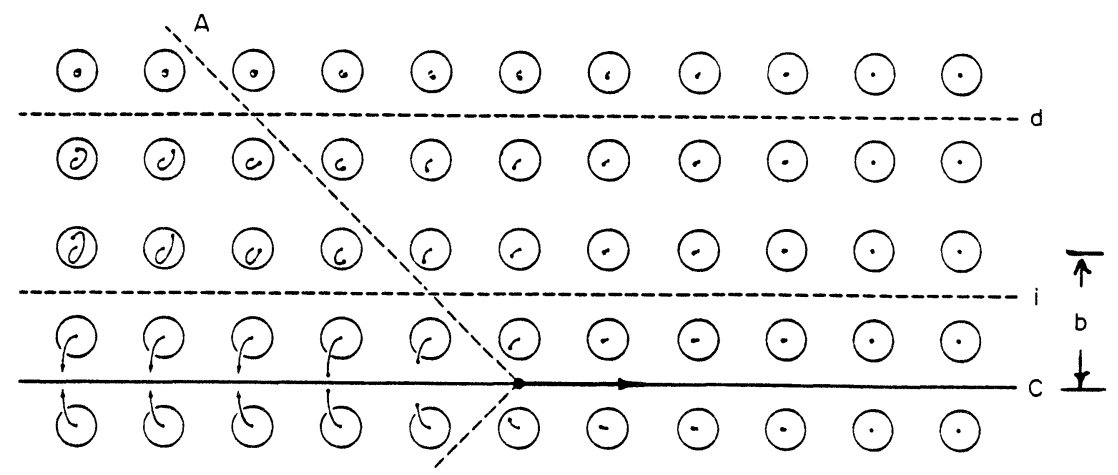

Fig. 10. A schematic representation of a charged particle passing through a solid. The polarization induced in the constituent atoms is indicated by the arrows, and the impact parameter with respect to a given row of atoms is shown (Bohr, 1948). 
media. It is also known to atomic theorists as the "Mott impact parameter".

Electrons that are loosely bound in isolated atoms may become nearly free in real condensed matter composed of these atoms, and may extend over macroscopic dimensions. In this case, $\omega_{o}$ becomes very small or zero for such valence electrons. However, screening comes into play here and the cutoff parameter is to be computed by replacing $\omega_{o}$ by $\omega_{p}$, the plasma frequency for these electrons. Note that even when all electrons are relatively well-localized, as in an insulator, Coulomb forces couple atoms of the assembly, as in the schematic derivation of equations (9)-(17), and excitations may be extended in space. Excitations created by a swift charged particle in a piece of condensed matter may be coherent over large distances and may constitute a model that can affect the way in which excitations, or damage, are distributed in space and time following the passage of an electron or an ion.

\subsection{Theory}

The problem of visualizing quantal collisions in the space (and perhaps time) variable has been faced by Bohr (1948), Williams (1945) and by Neufeld (1953), among others. Fano (1970) has pointed out that in condensed media, excitations may be coherent over distances comparable with $b_{c}$ and thus may involve the collective motion of $\sim 10^{6}$ atoms. He has noted the lack of a comprehensive theory of the impact parameter representation of collisions in condensed natter and has described a preliminary approach to uch a theory. All of the approaches made to this roblem to date involve the assumption that atoms or nolecules in the system are weakly interacting with ne another.

Below we represent the interaction probability in erms of the dielectric response function and the usual nomentum transfer variable, as transformed into a unction of space by several alternative methods. The esulting transforms are compared. We also consider he probability of decay of the initially unlocalized coherent excitations into localized ones.

3.2.1. The inverse mean free path. We rewrite equation (22) in terms of $\hbar \kappa$, the momentum transfer perpendicular to the velocity of the ion, as

$$
\frac{\mathrm{d}^{3} \Lambda^{-1}}{\mathrm{~d} \omega \mathrm{d}^{2} \kappa}=\frac{Z^{2} e^{2}}{\pi^{2} \hbar} \frac{1}{v^{2}\left(\kappa^{2}+\omega^{2} / v^{2}\right)} \operatorname{Im}\left[\frac{-1}{\epsilon_{k, \omega}}\right] .
$$

This is the differential inverse mean free path (DIMFP) for energy loss, $\hbar \omega$, and momentum transfer, $\hbar \kappa$, to condensed matter characterized by the dielectric function $\epsilon_{k, \omega}$. The magnitude of the total momentum $\hbar k=\hbar\left(\kappa^{2}+\omega^{2} / v^{2}\right)^{1 / 2}$.

The goal here is to express the DIMFP in terms of a spatial variable that we will interpret as impact parameter, rather than the momentum transfer, $\hbar \kappa$.

3.2.2. The "Van Hove transform". Van Hove (1954) showed that the first Born approximation cross section of matter for incident neutrons may be factored into a product of functions, one of which contains all of the dependence on the properties of the matter. This function, called the pair distribution function, contains the scattering properties of the system, and depends on energy and momentum transfer to it. When Fourier-transformed into a function, $G(\mathbf{r}, t)$, of space and time, this was interpreted by Van Hove as describing the quantal average density distribution at $\mathbf{r}$, and $t$, if a particle is located at the origin of coordinates at $t=0$. Van Hove notes that $G(\mathbf{r}, t)$ is a complex function because it reflects quantum properties of the system. It has been used extensively in analyzing the properties of dense gases and liquids as inferred from neutron-scattering experiments.

Pines and Nozieres (1966) state that the comparable quantity in a polarizable system characterized by the dielectric function $\epsilon_{k, \omega}$, is to be interpreted as the function describing correlations between density fluctuations at different space-time points. Rather than dealing with the correlation function $G$ as defined by Van Hove, we proceed to compute the two-dimensional Fourier transform of the DIMFP in order to compare directly with other forms described below. Thus

$$
\begin{aligned}
\Lambda_{\mathrm{vH}}^{-1}(b)=\left(2 Z^{2} e^{2} / \hbar v^{2}\right) \int_{0}^{\infty} \mathrm{d} \omega \int \mathrm{d}^{2} \kappa J_{o}(b \kappa) \\
\times \operatorname{Im}\left(-1 / \epsilon_{k, \omega}\right) /\left(\kappa^{2}+\omega^{2} / v^{2}\right),
\end{aligned}
$$

where we have integrated over $\omega$ in order to obtain an easily surveyed result. In Fig. 11 we have plotted $\Lambda_{\mathrm{VH}}^{-1}(b)$ vs $b$ for an ion with speed $v=20$ a.u. (e.g. a $10 \mathrm{MeV}$ proton), moving in an electron gas having the plasmon energy of $15.4 \mathrm{eV}$ as the solid line. In computing this result, we have assumed that the plasmon has negligible damping and quadratic dispersion with a cutoff at $k_{c}=\omega_{p} / v_{\mathrm{F}}$, where $v_{\mathrm{F}}$ is the Fermi speed for this electron gas. That is, we take

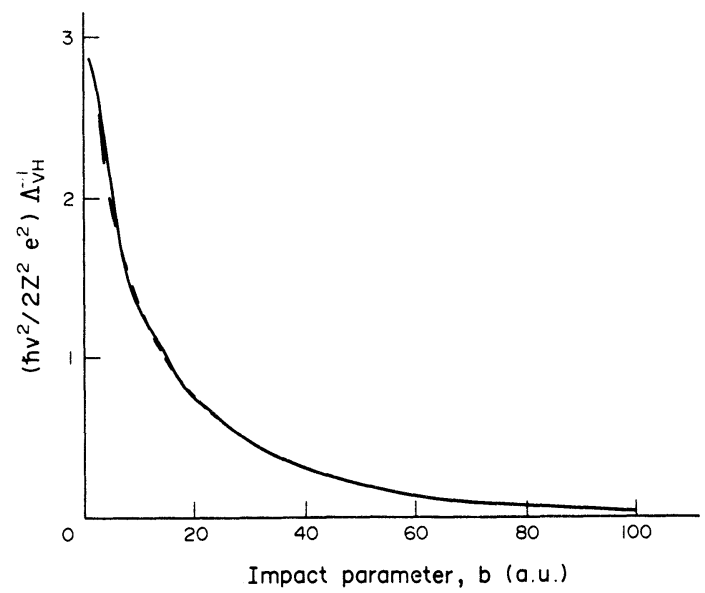

Fig. 11. A plot of the "Van Hove" inverse mean free path as a function of impact parameter for an ion with speed $v=20$ a.u. moving in an electron gas with a plasma energy of $15.4 \mathrm{eV}$. The dashed line shows a plot of the distribution computed from the approximate form [equation (32)] while the solid curve was calculated from equation (31). 
$\operatorname{Im}(-1 / \epsilon)=\left(\pi \omega_{p}^{2} / 2 \omega_{k}\right) \delta\left(\omega-\omega_{k}\right) \theta\left(k_{e}-k\right)$, where $\omega_{k}$ is given immediately following equation (8).

A simple and easily surveyed result is obtained if the plasmon is approximated as a dispersionless resonance at $\omega=\omega_{p}$ and if the integral over $\kappa$ is extended to infinity. Then

$$
\Lambda_{\mathrm{vH}}^{-1}(b) \approx \frac{2 \pi Z^{2} e^{2} \omega_{p}}{\hbar v^{2}} K_{o}\left(\omega_{p} b / v\right)
$$

where $K_{o}$ is the modified Bessel function of the second kind and order zero. For large $x$, $K_{o}(x) \sim \exp (-x) /(2 \pi x)^{1 / 2}$.

In Fig. 11 we have plotted $\Lambda_{\mathrm{VH}}^{-1}(b)$ vs $b$ calculated from equation (32) as the dashed line for the same conditions as for that computed from equation (31). In obtaining this result we have assumed that the plasmon has negligible damping and quadratic dispersion with a cutoff at $k_{c}=\omega_{p} / v_{\mathrm{F}}$.

3.2.3. The Chang-Raman transform. An approach to the problem of expressing quantal probabilities in terms of an impact parameter variable has been advocated by Fano (1970), based on a method introduced into high-energy physics by Chang and Raman (1969). To apply this method to the DIMFP of equation (30) we first integrate over all $\omega$ to find that

$$
\begin{aligned}
\mathrm{d}^{2} \Lambda_{\mathrm{CR}}^{-1} / \mathrm{d}^{2} \kappa=\left(\frac{Z^{2} e^{2}}{\pi^{2} \hbar v^{2}}\right) & \int_{0}^{\infty} \frac{\mathrm{d} \omega}{\kappa^{2}+\omega^{2} v^{2}} \\
& \times \operatorname{Im}\left[\frac{-1}{\epsilon_{k, \omega}}\right] \equiv|\sigma(\kappa)|^{2} .
\end{aligned}
$$

We now seek to eliminate $\kappa$ in favor of a spatial variable that we will interpret as an impact parameter. Thus

$$
\begin{aligned}
\Lambda_{\mathrm{CR}}^{-1}= & \int \mathrm{d}^{2} \kappa|\sigma|^{2} \\
= & \int \mathrm{d}^{2} \kappa \int \mathrm{d}^{2} \kappa^{\prime} \sigma(\boldsymbol{\kappa}) \sigma^{*}\left(\boldsymbol{\kappa}^{\prime}\right) \delta^{2}\left(\boldsymbol{\kappa}-\boldsymbol{\kappa}^{\prime}\right) \\
= & \frac{1}{(2 \pi)^{2}} \int \mathrm{d}^{2} \kappa \int \mathrm{d}^{2} \kappa^{\prime} \sigma(\boldsymbol{\kappa}) \sigma^{*}\left(\boldsymbol{\kappa}^{\prime}\right) \\
& \times \int \mathrm{d}^{2} b \exp \left(i \mathbf{b} \cdot\left[\boldsymbol{\kappa}-\boldsymbol{\kappa}^{\prime}\right]\right) \\
= & \frac{1}{(2 \pi)^{2}} \int \mathrm{d}^{2} b \mid\left.\int \mathrm{d}^{2} \kappa \exp (\text { i } \cdot \mathbf{b}) \sigma(\boldsymbol{\kappa})\right|^{2} .
\end{aligned}
$$

The integrand of equation (34) is now set equal to the DIMFP in impact parameter space, viz.,

$$
\begin{aligned}
\frac{\mathrm{d}^{2} \Lambda_{\mathrm{CR}}^{-1}}{\mathrm{~d}^{2} b}= & \frac{Z^{2} e^{2}}{4 \pi^{4} \hbar v^{2}} \mid \int \mathrm{d}^{2} \kappa \\
& \times\left.\exp (i \boldsymbol{\kappa} \cdot \mathbf{b})\left\{\int_{0}^{\infty} \frac{\mathrm{d} \omega}{k^{2}} \operatorname{Im}\left[\frac{1}{\epsilon_{k, \omega}}\right]\right\}^{1 / 2}\right|^{2} .
\end{aligned}
$$

One obtains a useful approximation to this equation for the case where the medium supports plasma oscillations codified in a dielectric function that is sharply peaked about a quadratic dispersion line having the form

$$
\omega_{k}=\left(\omega_{p}^{2}+\beta^{2} k^{2}\right)^{1 / 2}
$$

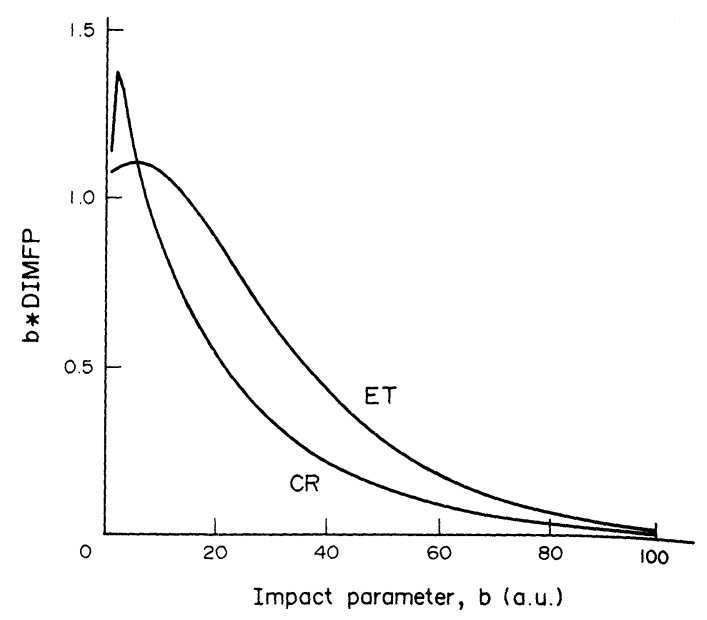

FIG. 12. A plot of the scaled DIMFP vs impact parameter $b$ computed from the Chang-Raman transform equation (37) (labeled CR), and from the energy transfer transform equation (38) after integration over $\omega$ (labeled ET). The plasma energy is taken to be $10.2 \mathrm{eV}$. Both sets of data have been multiplied by $b$ to emphasize the differences for large $b$.

as indicated above for the hydrodynamical model. In this case one may show that

$$
\begin{aligned}
p_{\mathrm{CR}}(b) \equiv & \frac{\mathrm{d}^{2} \Lambda_{\mathrm{CR}}^{-1}}{\mathrm{~d}^{2} b} \\
\cong & \left(\frac{Z e}{\beta v}\right)^{2} \frac{\omega_{p}^{3}}{\pi \hbar} I_{o}^{2}\left(\frac{b \omega_{p}}{\sqrt{ } 2 \beta}\left[1-\frac{\sqrt{ } 2 \beta}{v}\right]^{1 / 2}\right) \\
& \times K_{o}^{2}\left(\frac{b \omega_{p}}{\sqrt{ } 2 \beta}\left[1+\frac{\sqrt{2 \beta}}{v}\right]^{1 / 2}\right) .
\end{aligned}
$$

This function is plotted in Fig. 12, as the line labeled $\mathrm{CR}$, for the same conditions assumed in plotting Fig. 11, except that $\hbar \omega_{p}=10 \mathrm{eV}$.

The Chang-Raman transform does not seem to be useful for finding the DIMFP expressed as a function of both $\omega$ and impact parameter. It appears that when the DIMFP has a narrow resonance in the $k-\omega$ plane such as may occur when plasma oscillations exist in the medium, the transformation to the $b$ variable gives an indeterminate result.

3.2.4. The energy transfer transform. It is possible to factor the integrand of equation (30) in such a way that the DIMFP may be written as a function of energy as well as impact parameter. Writing

$$
\begin{aligned}
\frac{\mathrm{d} \Lambda_{\mathrm{ET}}^{-1}}{\mathrm{~d} \omega}= & \frac{1}{\hbar}\left(\frac{Z e}{\pi v}\right)^{2} \int \frac{\mathrm{d}^{2} \kappa}{k^{2}} \operatorname{Im}\left[\frac{-1}{\epsilon_{k, \omega}}\right] \\
= & \frac{1}{\hbar}\left(\frac{Z e}{2 \pi^{2} v}\right)^{2} \int \mathrm{d}^{2} b \int \mathrm{d}^{2} \kappa \int \mathrm{d}^{2} \kappa^{\prime} \\
& \times \exp \left[i \mathbf{b} \cdot\left(\boldsymbol{\kappa}-\boldsymbol{\kappa}^{\prime}\right)\right] \frac{\mathbf{k} \cdot \mathbf{k}^{\prime}}{k^{2} k^{\prime 2}} \operatorname{Im}\left[\frac{-1}{\epsilon_{k, \omega}}\right],
\end{aligned}
$$

so that

$$
p_{\mathrm{ET}}(b) \equiv \frac{\mathrm{d}^{3} \Lambda_{\mathrm{ET}}^{-1}}{\mathrm{~d} \omega \mathrm{d}^{2} b}=\frac{Z^{2} e^{2} \omega}{\pi^{2} \hbar v^{3}} \int_{0}^{\infty} \frac{\kappa \mathrm{d} \kappa}{\left(\kappa^{2}+\omega^{2} / v^{2}\right)}
$$




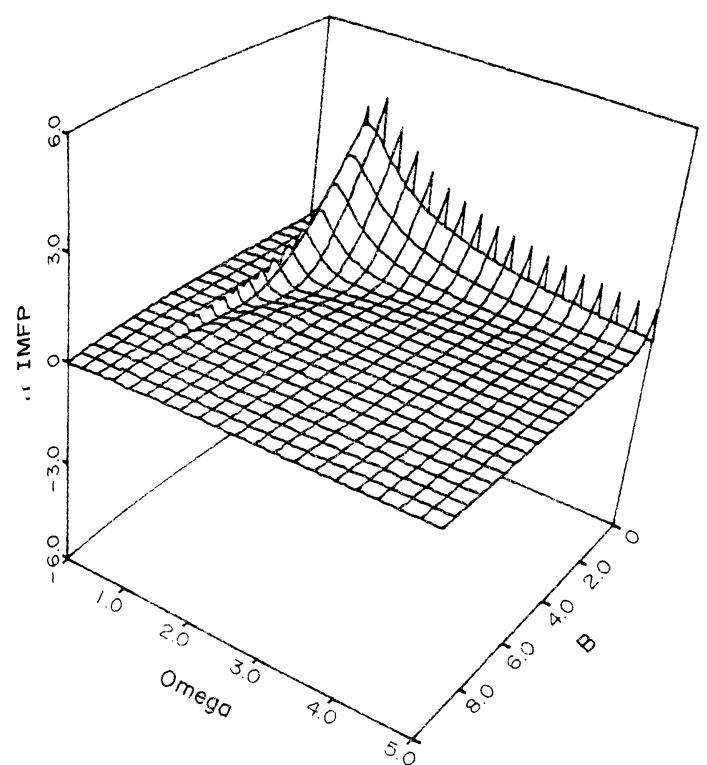

FIG. 13. The DIMFP for an electron gas, plotted as a function of impact parameter $b$ and energy transfer $\hbar \omega$. The DIMFP is normalized arbitrarily, and $b$ and $\hbar \omega$ are expressed in a.u. An approximate expression for $\epsilon_{k, \omega}$ has been used in this calculation: the particle is assumed to have a speed of 20 a.u. and $\hbar \omega_{p}=15.4 \mathrm{eV}$. This is equivalent to a proton with an energy of $10 \mathrm{MeV}$.

$$
\begin{aligned}
& \times\left[\frac{\omega}{v} K_{o}\left(\frac{b \omega}{v}\right) J_{o}(\kappa b)\right. \\
& \left.+\kappa K_{1}\left(\frac{b \omega}{v}\right) J_{1}(\kappa b)\right] \operatorname{Im}\left[\frac{-1}{\epsilon_{k, \omega}}\right],
\end{aligned}
$$

interpreting the integrand as before. We term this the "energy transfer transform" since it agrees precisely with the formula obtained by computing the energy transferred to the medium at fixed impact parameter, using semi-classical dielectric theory, and then dividing the integrand in the $\omega$ variable by $\hbar \omega$.

An equation comparable with equation (37) is obtained by integrating equation (38) over $\omega$. The solid line labeled ET in Fig. 12 shows numerical results for the conditions specified for Fig. 11. The curve labeled CR was computed from equation (37). One finds that $p_{\mathrm{ET}}(b)$ has a rather different dependence on $b$ than does $p_{\mathrm{CR}}(b)$. Their asymptotic forms are

and

$$
p_{\mathrm{CR}} \sim \exp \left(-2 \omega_{p} b / v\right) / b^{2}
$$

$$
p_{\mathrm{ET}} \sim \exp \left(-2 \omega_{p} b / v\right) / b .
$$

As we show below, $p_{\mathrm{ET}}(b)$ corresponds more nearly to the spatial variation of the localization probability than does $p_{\mathrm{CR}}(b)$.

In Fig. 13 the function of equation (38) is plotted in the $b-\omega$ plane. A simplified representation of the dielectric function of the medium was used in obtaining this plot. It shows the DIMFP for a charged particle with a velocity of 4 a.u. proceeding through an electron gas with plasma energy of $15.4 \mathrm{eV}$ and includes contributions from single-electron transitions as well as plasmons. The plasmon contribution appears as a rather broad resonance in $\omega$, peaking at near the plasma energy for $b>2$ a.u., but showing a ridge concentrated at very small $b$ and extending to large values of $\omega$. This corresponds to the well-known fact that encounters at small impact parameter tend to involve large energy transfers to the medium. This feature might be called the "Bohr ridge" in analogy with the Bethe ridge of the DIMFP in the $k-\omega$ plane.

It is interesting that although this function is always real, unlike the Van Hove correlation function, it may be negative over small regions of the $b-\omega$ plane. An example of this may be seen in the vicinity of the point $\omega \sim 0.7$ a.u. and $b \sim 5$ a.u. in Fig. 13 . This is an indication of the essential quantal character of the interactions under consideration. However, it should be noted that the DIMFP integrated over $\omega$, or over $b$, is positive definite.

\section{THE LOCALIZATION OF INITIALLY UNLOCALIZED EXCITATIONS}

The mathematical problem of representing interactions in condensed media in terms of an impact parameter-like variable is separate from, but related to, that of establishing the location and products of plasmon decay. The plasmon is thought to be an unlocalized excitation in the background of the valence electron gas of a piece of condensed matter. Equation (8) represents the plasmon in an electron gas as a plane wave extending over the whole volume of the medium. As indicated in Section 2.1, the electric field of a plasmon in an ideal electron gas is in the same direction as its propagation vector. The electric field of a photon in vacuum is perpendicular to its propagation vector, but otherwise these entities are similar. Frolich and Peltzer (1955) pointed out this similarity and Wolff (1953) noted that a longwavelength plasmon $\left(k \ll 1 \AA^{-1}\right)$ should decay with an absorption coefficient (IMFP) equal to that of a photon with the same frequency in the same medium [equation (23)]. This equality should be well-satisfied in real condensed matter where plasmons have energies $\leqslant 30 \mathrm{eV}$. In this range, photon-induced electronic transitions are of dipole character; the wavelength of such photons is $\geqslant$ several hundred angstroms which is much greater than the wavelength of the electron produced.

In the idealized electron gas (jellium), a plasmon may decay only to a multi-quasiparticle final state. Decay into a single electron-hole pair is forbidden by energy-momentum conservation considerations. The plasmon generally carries insufficient momentum to excite a real plasmon. Glick and Long (1971) have evaluated approximately the rate of decay of a plasmon in jellium into a two-quasiparticle final state for a range of electron gas densities.

The alkali metals are nature's closest approximations to the electron gas. Photon (and hence 


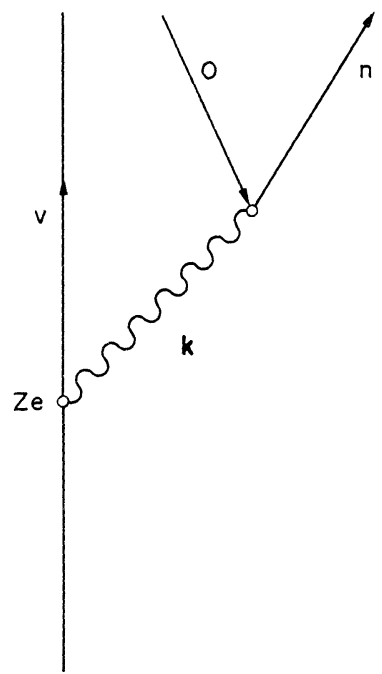

FIG. 14. A Feynman diagram representing the process of virtual plasmon creation by a swift charged particle in condensed matter followed by excitation of a real, single electron-hole pair in the medium.

plasmon) absorption in these solids has been treated theoretically (Animalu, 1970; Mahan, 1967) in the nearly-free-electron-gas model. Here the ion cores of the solid are represented by pseudopotentials on which a conduction electron, excited to a virtual state by a photon, may scatter. Such scattering permits excited electrons to lose momentum to the solid so that absorption of a photon may occur.

In this approximation the probability of plasmon (photon) localization at a point in space is proportional to the square of the plasmon (photon) electric field strength at that point. It seems clear that this should be true irrespective of the nature of the solid as long as the plasmon wavelength is much less than that of the electron, i.e. $k_{\text {plasmon }} \ll 1 \AA^{-1}$.

We may go beyond the long-wavelength approximation in a simple model of the localization process. Assume that an impurity site in a condensed medium is occupied by an electron in an orbital $u_{o}(\mathbf{r})$, situated at $\mathbf{r}$. Let a swift ion with speed $v$ traverse the medium at impact parameter $b$ relative to the impurity. If the eigenenergies and wave functions of the impurity site electron are $\hbar \omega_{n}$ and $u_{n}(\mathbf{r})$, then the probability $P_{n}$ that the electron is excited through virtual collective states in the medium from its ground state to the $n$th excited state is illustrated by the Feynman diagram of Fig. 14. $P_{n}$ may be written

$$
\begin{aligned}
P_{n}=\left(\frac{Z e}{\pi \hbar v}\right)^{2} \mid & \frac{\mathrm{d}^{2} \kappa}{k^{2}} \\
& \times\left.\exp (i \boldsymbol{\kappa} \cdot \mathbf{b})\left\langle n\left|e^{i \mathbf{k} \cdot \mathbf{b}}\right| o\right\rangle\left(\frac{1}{\epsilon_{k, \omega_{n o}}}\right)\right|^{2},
\end{aligned}
$$

where $\omega_{n o}=\omega_{n}-\omega_{o}, k^{2}=\kappa^{2}+\omega_{n o}^{2} / v^{2}$, and where it is understood that the integration over $\kappa$ is to include only the region of $(k, \omega)$ space corresponding to collective states of the medium.

Equation (39) has been evaluated for illustrative purposes assuming $u_{o}(\mathbf{r})$ and $u_{1}(\mathbf{r})$ are Slater orbitals

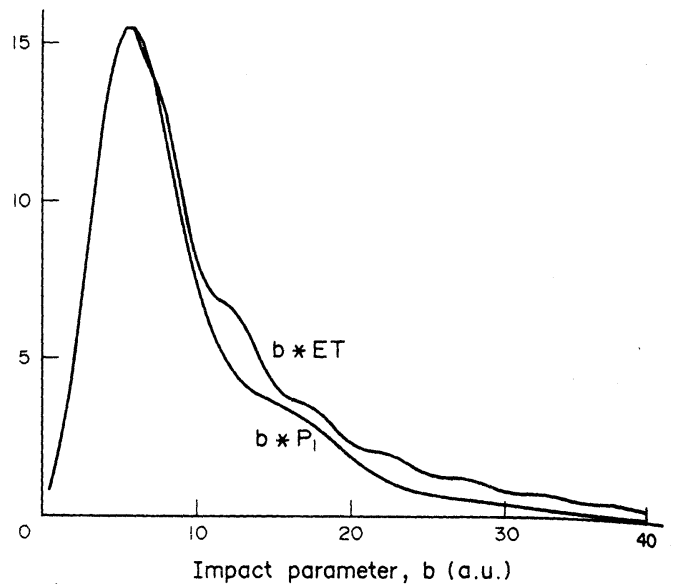

FIG. 15. The excitation probability, $P_{1}$, of an electron bound to an impurity site, plotted as a function of the impact parameter of the site relative to the trajectory of a charged particle with speed 20 a.u. For comparison, the curve labeled "ET" is computed from equation (38) after integration over $\omega$ and represents the DIMFP $\mathrm{d}^{2} \Lambda_{\mathrm{ET}}^{-1} / \mathrm{d}^{2} b$ for the same particle. Arbitrary normalization of both curves has been used. Both sets of data have been multiplied by $b$ for emphasis.

and have $s$ - and $p$-wave character, respectively. Figure 15 shows a plot of the dependence of $P_{1}$ on $b$, taking the excitation energy of the electron to be $18.8 \mathrm{eV}$. On the same plot is shown the energy transfer transform vs $b$, computed from the integral of equation (38) over $\omega$ and labeled "ET". The medium is taken to be an electron gas with $\hbar \omega_{p}=15.4 \mathrm{eV}$, described by the Mermin (1970) dielectric function for a damping constant of $3.8 \mathrm{eV}$ and a cutoff wave number $k_{c}=\omega_{p} / v_{\mathrm{F}}$.

Ritchie and Brandt (1975) and Brandt and Ritchie (1973) have discussed qualitatively the effect of plasmon propagation and damping on the localization process. They conclude that these are rather unimportant and that, to a good approximation, the impact parameter $b_{c} \approx v / \omega_{p}$ characterizes the decrease of the localization probability with increasing impact parameter. Calculations made with equation (39) confirm these conclusions.

\section{THE ELECTRON MONTE CARLO CODE, OREC}

We have developed a Monte Carlo code, OREC, for determining the transport of electrons and ions in liquid water (Hamm et al., 1975, 1978a,b, 1985; Ritchie et al., 1978; Turner et al., 1978, 1983; Paretzke et al., 1986). Ritchie et al. (1978) have described the procedure used to esitmate DIMFPs for charged particles in water from experimental data on $\epsilon(\omega)$, the long-wavelength dielectric function of water.

In addition to utilizing actual experimental data on liquid water, the principal non-scaling effect that we have included in OREC is the localization of initially non-localized excitations created in the medium by 


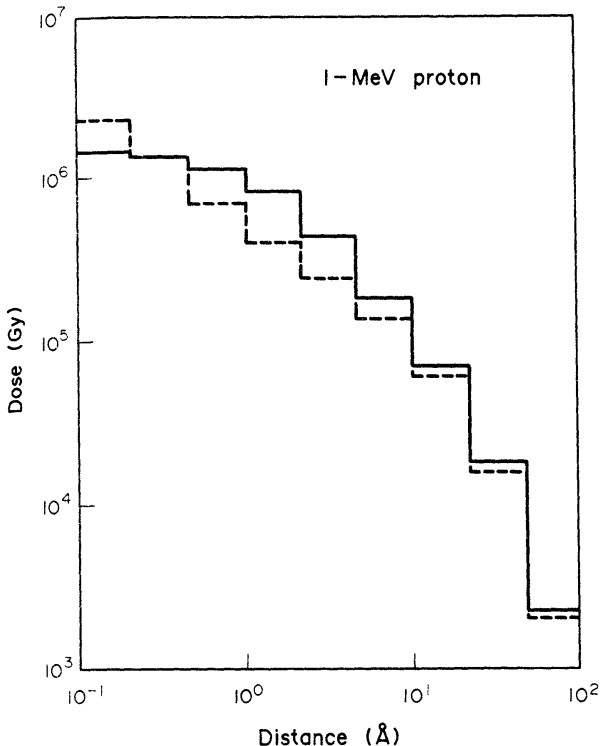

FIG. 16. The radial dose vs distance from the track of a $1 \mathrm{MeV}$ proton in liquid water computed from the code OREC. The solid histogram shows results found using the algorithm described in the text for modeling the generation and decay of collective excitations. The dashed histogram was obtained by omitting this algorithm.

swift particles. In OREC, electrons are transported in the standard manner by choosing flight path lengths stochastically, using tables of MFPs as a function of electron energy (Hamm et al., 1978a, b). At a collision locus the kind of interaction is chosen using probability tables of the various interactions, e.g. elastic, ionizing, excitational, etc. If the interaction is inlastic and corresponds to an energy loss lying in the teighborhood of the assumed plasmonic resonance in $\mathrm{n}\left(-1 / \epsilon_{k, \omega}\right)$, taken to extend from $\hbar \omega=7.4$ to $50 \mathrm{eV}$, then it is assumed that a collective state with lateral extension $\sim v / \omega$ has been created. On this basis a localization site is chosen laterally from a distribution characterized by the length $\sim v / \omega$, with the general form of equation (38). The type of localized transition resulting from the decay of the collective state is found from the set of values of

$$
\int_{k_{-}}^{k_{+}} \frac{\operatorname{Im}\left(\epsilon_{k, \omega}^{i}\right) \mathrm{d} k}{k\left|\epsilon_{k, \omega}\right|^{2}}
$$

where $k_{ \pm}=\left(2 m E / \hbar^{2}\right)^{1 / 2} \pm\left[2 m(E-\hbar \omega) / \hbar^{2}\right]^{1 / 2}$ and $E$ is the electron energy. Here $\epsilon_{k, \omega}^{i}$ is the partial dielectric function for the $i$ th kind of transition. If the transition is an ionizing one, the resulting electron is transported in the same manner and the generation of histories is continued in the standard Monte Carlo approach to obtain estimates of desired physical end points.

Some results from an OREC calculation of the radial dose expressed in Grays $\left(10^{4} \mathrm{erg} / \mathrm{g}\right)$ as a function of distance from the track of a $1 \mathrm{MeV}$ proton in liquid water, are shown in Fig. 16. The calculations were made with (solid histogram) and without (dashed histogram) use of the algorithm for modeling the generation and localization of collective excitations. In Fig. 17 one sees the effect of including, or omitting, this algorithm on the $G$-values for $\mathrm{OH}$ formation and for generation of the hydrated electron as a function of time after a $5 \mathrm{keV}$ electron traverses a water medium. It is perhaps not surprising that the effects of plasmon generation and decay are small in this case; the Bohr impact parameter corresponding to the velocities involved here is only a few angstroms. One would expect the effects in question to be important for high velocity, large atomic number ions.

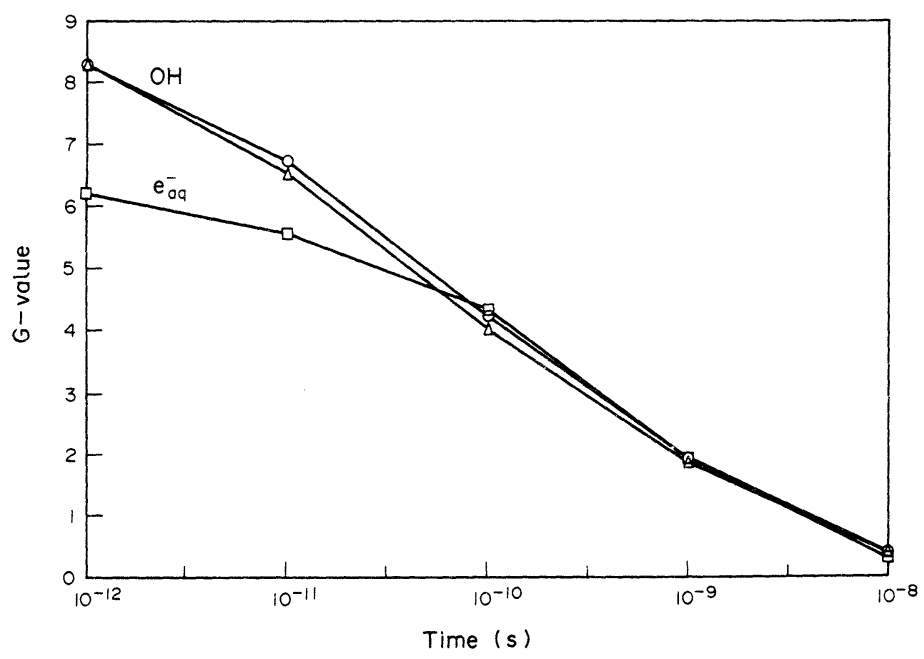

FIG. 17. $G$-values for $\mathrm{OH}$ formation and generation of the hydrated electron in water as a function of time after irradiation by a $5 \mathrm{keV}$ electron. The $\mathrm{OH}$ data shown by the symbol $(O)$ were found using OREC with the algorithm for generation and decay of collective excitations while the symbol $(\triangle)$ shows data obtained without using this algorithm. The data for $e_{\mathrm{aq}}^{-}$were found to be insensitive to whether the algorithm is used or not. 


\section{CONCLUSIONS}

We have described some of the properties of plasmons in condensed matter. Several different impact parameter representations of these coherent excitations were considered and compared numerically. Decay of the plasmon into a single-particle state was considered and evaluated on the basis of a simple model. The effect of such decay on end-point calculations in our code OREC was evaluated.

\section{DEDICATION}

This paper is dedicated to Dr Robert Katz, who has made outstanding contributions to track physics over many years. His enthusiasm, his originality, the freshness of his ideas and the appealing physical concepts that underlie them have impressed us all. One of the most outstanding aspects of Bob's remarkable personality is his boundless good nature and gusto for life. He responds to both praise and criticism with high good humor and wit. It has been a privilege to know and work with Bob and to count him as a colleague and friend. We hope that he will continue to do research in this area of physics for many, many years.

Acknowledgements -Conversations with Robert Katz and Herwig Paretzke were very helpful in this connection.

\section{REFERENCES}

Animalu A. O. E. (1970) Many-electron effects in the optical conductivity of simple metals by the Kubo formula. Phys. Rev. B2, 282-289.

Ashley J. C. and Anderson V. E. (1981) Interaction of low-energy electrons with silicon dioxide. J. Electron Spectrosc. rel. Phenom. 24, 127-148.

Ashley J. C. (1988) Interaction of low-energy electrons with the electron beam resist poly(butene-1-sulfone). J. appl. Phys. 63, 4620-4625 (and references therein).

Bethe H. A. (1930) Auf Theorie des Durchgangs schneller Korpuskularstrahlen durch Materie. Annln Phys. 5, 325-400.

Bohr N. (1913) On the decrease of velocity of swiftly moving electrified particles in passing through matter. Phil. Mag. 25, 10-31.

Bohr N. (1948) The penetration of atomic particles through matter. K. danske Vidensk. Selsk. Skr. 18, 1-144.

Bloch F. (1933) Bremsvermogen von Atomen mit mehrer Elektronen. Z. Phys. 81, 363-376.

Brandt W. and Ritchie R. H. (1973) Primary processes in the physical stage. In Proc. Conf. Phys. Mech. Radiat. Biol., pp. 20-46. Natn. Tech. Info. Service, U.S. Dept. of Commerce, Springfield, VA 22151, U.S.A. CONF721001 .

Chang N. P. and Raman K. (1969) Impact parameter representation and the coordinate space description of a scattering amplitude. Phys. Rev. 181, 2048-2055.

Daniels J., Festenberg C. v., Raether H. and Zeppenfeld K. (1970) Optical constants of solids by electron spectroscopy. Springer Tracts mod. Phys. 54, 77-135.

Ehrenreich H. and Cohen M. H. (1959) Self-consistent field approach to the many-electron problem. Phys. Rev. 115, 786-790.

Ehrenreich H. and Philipp H. R. (1962) Optical properties of semiconductors in the ultra-violet. In Proc. Int.
Conf. Phys. Semiconductors (Edited by Strickland A. C.), pp. 367-374. Bartholomew Press, Dorking, U.K.

Fano U. (1960a) Normal modes of a lattice of oscillators with many resonances and dipolar coupling. Phys. Rev. 118, $451-455$.

Fano U. (1960b) Collective effects in absorption of energy from ionizing radiation. In Comparative Effects of Radiation (Edited by Burton E., Kirby-Smith J. and Magee J.), pp. 14-21. Wiley, New York.

Fano U. (1970) The formulation of track structure theory. In Charged Particle Tracks in Solids and Liquids, pp. 1-7. The Institute of Physics and the Physical Society, Conf. Series No. 8, London.

Frolich H. and Peltzer H. (1955) Plasma oscillations and the energy loss of charged particles in solids. Proc. phys. Soc. A58, 525-530.

Glick A. J. and Long W. F. (1971) High-frequency damping in a degenerate electron gas. Phys. Rev. B4, 3455-3460.

Hamm R. N., Wright H. A., Ritchie R. H., Turner J. E. and Turner, T. P. (1975) Monte Carlo calculation of transport of electrons through liquid water. In Proc. 5th Symp. Microdosim., pp. 1037-1053. EUR 5452 d-e-f.

Hamm R. N., Wright H. A., Katz R., Turner J. E. and Ritchie R. H. (1978a) Calculated yields and slowingdown spectra for electrons in liquid water: implications for electron and photon RBE. Physics Med. Biol. 23, $1149-1161$.

Hamm R. N., Wright H. A., Turner J. E. and Ritchie R. H. (1978b) Spatial correlation of energy deposition events in irradiated liquid water. In 6th Symp. Microdosim. (Edited by Boag J. and Ebert H. G.), pp. 179-186. Harwood, London.

Hamm R. N., Turner J. E, Ritchie R. H. and Wright H. A. (1985) Calculation of heavy-ion tracks in liquid water. Radiat. Res. 104, S-20-S-26.

Heller J. M. Jr, Hamm R. N., Birkhoff R. D. and Painter L. R. (1974) Collective oscillation in liquid water. $J$. chem. Phys. 60, 3483-3486.

Lindhard J. (1954) On the properties of a gas of charged particles. K. danske Vidensk. Selsk. Skr. 28(8), 1-56.

Mahan G. D. (1967) Interband transitions in sodium. Phys. Lett. 24, A708-710.

Mermin N. D. (1970) Lindhard dielectric function in the relaxation-time approximation. Phys. Rev. B1, 2362-2363.

Neufeld J. (1953) Energy-losses of charged particles of intermediate energy. Proc. phys. Soc. A66, 489-596.

Palik E. D. (1985) Handbook of Optical Constants of Solids. Academic Press, New York.

Paretzke H. G., Turner J. E., Hamm R. N., Wright H. A. and Ritchie R. H. (1986) Calculated yields and fluctuations for electron degradation in liquid water and water vapor. J. chem. Phys. 84, 3182-3188.

Pines D. and Bohm D. (1952) Collective description of electron interactions: II. Collective vs individual particle aspects of the interactions. Phys. Rev. 85, 338-355.

Pines D. and Nozieres P. (1966) The Theory of Quantum Liquids. W. A. Benjamin, New York.

Raether H. (1965) Solid state excitations by electrons. Springer Tracts mod. Phys. 38, 84-157.

Ritchie R. H. (1957) Plasma losses by fast electrons in thin films. Phys. Rev. 106, 874-881.

Ritchie R. H., Tung C. J., Anderson V. E. and Ashley J. C. (1975) Electron slowing-down spectra in solids. Radiat. Res. 64, 181-204.

Ritchie R. H. and Brandt W. (1975) Primary processes and track effects in irradiated media. In Radiation Research; Biomedical, Physical, and Chemical Perspectives, pp. 315-324. Academic Press, New York. 
Ritchie R. H., Hamm R. N., Turner J. E. and Wright H. A. (1978) The interaction of swift electrons with liquid water. Proc. 6th Symp. Microdosim., Brussels, May 1978, Vol. I, pp. 179-186. EUR 6064 d-e-f.

Rosenfeld L. (1965) Theory of Electrons, Chap. V. Dover, New York.

Sramek S. J. and Cohen M. L. (1972) Frequency- and wave-vector-dependent dielectric function for $\mathrm{Ge}$, GaAs, and ZnSe. Phys. Rev. B6, 3800-3804.

Tonks L. and Langmuir I. (1929) Oscillations in ionized gases. Phys. Rev. 33, 195-210.

Tung C. J., Ashley J. C., Birkhoff R. D. and Ritchie R. H. (1977) Electron slowing-down flux spectrum in $\mathrm{Al}_{2} \mathrm{O}_{3}$. Phys. Rev. B16, 3049-3055.

Turner J. E., Hamm R. N., Wright H. A. and Ritchie R. H. (1978) Calculated electron slowing-down spectra for liquid water irradiated by X-and gamma rays-implications for photon RBE. In 6th Symp. Microdosim. (Edited by Boag J. and Ebert H. G.), pp. 375-382. Harwood, London.
Turner J. E., Paretzke H. G., Hamm R. N., Wright H. A. and Ritchie R. H. (1983) Collective aspects of charged particle track structure in non-metallic solids. In Proc. 8th Symp. Microdosim. Jülich, F.R.G., 1982, pp. 145-152. EUR 8395 EN.

Van Hove L. (1954) Correlations in space and time and Born approximation scattering in systems of interacting particles. Phys. Rev. 95, 249-262.

Walter J. P. and Cohen M. L. (1972) Frequency- and wave vector-dependent dielectric function for silicon. Phys. Rev. B5, 3101-3110.

Williams E. J. (1945) Space-time concepts in collision problems. Rev. mod. Phys. 17, 217-245 (and references therein).

Williams M. W., Hamm R. N., Arakawa E. T., Painter L. R. and Birkhoff R. D. (1975) Collective electron effects in molecular liquids. Int. J. Radiat. Phys. Chem. 7, 95-108.

Wolff P. A. (1953) Theory of plasma waves in metals. Phys. Rev. 92, 18-23. 